\title{
Analysis of a compact size second order composite right/left handed coupled resonator band pass filter for wireless applications
}

\section{Original \\ Article}

\author{
Ahmed A. Ibrahim and Hesham F. A. Hamed
}

Faculty of Engineering, Minia University, El-Minia, Egypt

\begin{abstract}
Key words:
CRLH-TL, band pass filter, coupled resonator filter, second order filter
\end{abstract}

Corresponding Author: Ahmed A. Ibrahim Faculty of Engineering, Minia University, El-Minia-Egypt, E-mail: Ahmedabdel monem@mu.edu.eg,hfah66@yahoo.com, Tel.: 01006956456

\begin{abstract}
A microstrip compact size second order bandpass filter for wireless applications is presented. The band pass filter is based on Composite Right/Left handed transmission line. The two resonators are designed using only one composite right left handed unit cell. The bandpass filter has the advantage of compactness (its size is only $2.3 \times 2.3 \mathrm{~cm}^{2}$ ). A second order band pass filter with centre frequency of $4.77 \mathrm{GHZ}$ is designed, simulated and measured. The filter has bandwidth of $200 \mathrm{MHz}$ and insertion loss lower than $1.8 \mathrm{~dB}$ is achieved within pass band. A good agreement between simulated and measured result was achieved.
\end{abstract}

\section{INTRODUCTION}

Microwave band pass filters play an important role in various $\mathrm{RF} /$ microwave circuits and systems. With the rapid development of microwave and millimeter wave communication systems, it greatly stimulates the demand on high performance bandpass filters with compact dimensions, low insertion loss, high attenuation in stopband and low cost ${ }^{[1]}$. A high-performance planar microwave filter is usually required to have good selectivity, good attenuation level in rejection bands and low insertion loss. The use of microwave filters with transmission zeros in certain frequencies are very convenient to achieve high performance and good selectivity ${ }^{[2]}$.

Left handed transmission line is characterized by anti-parallel phase and group velocities. Also, it has a nonlinear progressive phase $^{[3-5]}$. Thanks to these properties, compact microwave devices such as power dividers, filters, phase shifter and resonators have been introduced $^{[6-12]}$. The composite right/left-handed (CRLH) transmission line (TL) has drawn particular attention in designing filters. It is implemented by periodically loading a conventional TL with lumped element series capacitors and shunt inductors ${ }^{[14-16]}$. A practical transmission line (TL) approach LHM is referred as a composite right / left handed (CRLH) TL ${ }^{[17]}$. In the equivalent circuit model, right handed $(\mathrm{RH})$ parameters consist of the series inductor and the shunt capacitor of the hosting TL while the series capacitor and shunt inductor are corresponding to the loading left handed $(\mathrm{LH})$ elements ${ }^{[17]}$. It is seen that, at lower frequency, the RH elements, CR and LR, will be open circuited and short circuited, respectively. Thus, the CRLH unit cell equivalent circuit reduces to that of $\mathrm{LH}$ equivalent circuit and hence it acts as a nearly ideal LH TL. Similarly, at higher frequency, the CRLH unit cell equivalent circuit reduces to that of RH equivalent circuit and hence it acts as an ideal RH TL ${ }^{[17]}$. CRLH coupled filters have been introduced in ${ }^{[18,19]}$

In this paper compact size second order bandpass filter for wireless applications is presented. The band pass filter is based on Composite Right/Left handed transmission line. The two resonators are designed depend on coupling matrix extraction and external quality factor. Based on the coupling coefficient approach, the performance of the device is investigated analytically and experimentally. The effect of the feed line configuration on the filter response is investigated also. A second order band pass filters with centre frequency of $4.77 \mathrm{GHZ}$ is designed, simulated and measured. The design and performance evaluation of the proposed filter has been done using the electromagnetic full wave simulation employing the commercial CST Microwave Studio. A Good agreement between simulated and measured result was achieved.

\section{FILTER SYNTHESIS USING OPTIMIZATION TECHNIQUE}

The coupling coefficient between resonators and the external quality factors at the input and the output resonator of the Fig. 1 are extracted using optimization technique ${ }^{[20]}$. The transmission function S21 $\left(\omega^{\prime}\right)$ is given by ${ }^{[20]}$. 


$$
\begin{aligned}
& \mid S_{21}\left(\left.\omega^{\prime}\right|^{2}-\frac{1}{1+\varepsilon^{2} F_{N}^{2}\left(\omega^{\prime}\right)}\right. \\
& \varepsilon=\left[10^{R / 10}-1\right]^{-1 / 2} \\
& F_{N}\left(\omega^{\prime}\right)=\cosh \left(\sum_{n=1}^{N} \cosh ^{-1}\left(x_{n}\right)\right) \\
& x_{n}=\frac{\omega^{\prime}-1 / \omega_{n}^{\prime}}{1-\omega^{\prime} / \omega_{n}^{\prime}}
\end{aligned}
$$

A network of multiple coupled lossless resonators is shown in Fig.1. The coupling coefficient between resonators $\mathrm{i}$ and $\mathrm{j}$ is denoted by $\mathrm{Mij}=\mathrm{Mji}$. It is obvious that the voltage source with internal resistance $\mathrm{R} 1$ is excited the structure at the resonator 1. Also the load is considered a resistor $\mathrm{R} 2$ connected to resonator $\mathrm{N}$. The normalized angular frequency $\omega^{\prime}$ is related to $\omega o$ and the bandwidth $\Delta \omega$ by $^{[20]}$ :

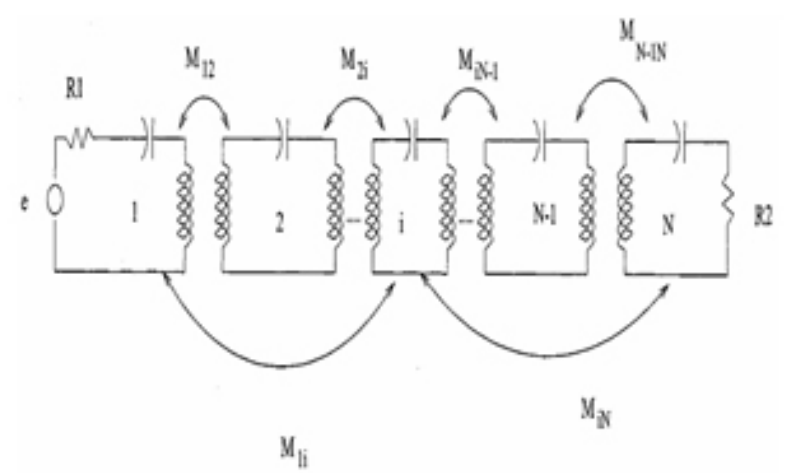

Fig. 1: Multiple coupled resonator band pass filter.

$$
\omega^{\prime}=\left(\omega_{0} / \Delta \omega\right)\left(\left(\omega / \omega_{0}\right)-\left(\omega_{0} / \omega\right)\right)
$$

After analysis, the scattering parameters are given by ${ }^{[20]}$ :

$$
\begin{aligned}
& S_{21}=2 \sqrt{R_{1} R_{2}} I_{N}=-2 j \sqrt{R_{1} R_{2}}\left[A^{-1}\right]_{N 1} \\
& S_{11}=1-2 R_{1} I_{1}=1+2 j R_{1}\left|A^{-1}\right|_{11}
\end{aligned}
$$

At this point, the synthesis problem can be formulated simply determine the coupling matrix $[\mathrm{M}]$ and the resistor $\mathrm{R} 1$ and $\mathrm{R} 2$ this problem can be solved using optimization. The optimization technique is formulated as minimization of a scalar cost function because it represents the difference between the performance achieved at any stage and the desired specifications. In the case of a microwave filter, the formulation of cost function may involve the specified and achieved values of the insertion loss and the return loss in the pass band, and the rejection in the stop band, so Filter characteristics obtained from the analysis are compared with the given specifications. If the results fail to satisfy the desired specifications, the designable (optimization) parameters of the filter are altered in a systematic manner. The sequence of filter analysis, comparison with the desired performance, and modification of designable parameters is performed iteratively until the optimum performance of the filter is achieved ${ }^{21}$. The cost function is given by ${ }^{[20]}$.

$$
\begin{aligned}
& K-\sum_{i=1}^{N} \mid s_{11}\left(\left.\left.\omega_{z i}\right|^{2}\right|^{2}+\sum_{i=1}^{D} \mid s_{21}\left(\left.\omega_{p_{1}}\right|^{2}+\left(\mid s_{11}\left(\omega--1 \mid-\frac{\delta}{\sqrt{1+\delta^{2}}}\right)^{2}\right.\right.\right. \\
& +\left(\mid s_{11}\left(\omega^{\prime}-1 \mid-\frac{\varepsilon}{\sqrt{1+\varepsilon^{2}}}\right)^{2}: 1\right.
\end{aligned}
$$

$\omega_{z i}^{\prime}$ and $\omega_{p i}^{\prime}$ are the zeros and poles of the filtering function $\mathrm{FN}$ it assumed to has $\mathrm{P}$ poles and $\mathrm{N}$ zeros respectively.

\section{REALIZATION OF THE CRLH COUPLED RESONATOR FILTER}

In this section we introduce the design and realization of the second order CRLH coupled resonator band pass filter. The filter specification is a bandwidth return loss $=10 \mathrm{~dB}, \mathrm{BW}=200 \mathrm{MHz}$, and centre frequency $\mathrm{f}_{\mathrm{o}}=4.77 \mathrm{GHz}$. Hence, the fractional bandwidth (FBW) is $\mathrm{BW} / \mathrm{f}_{\mathrm{o}}=0.041$. The proposed filter synthesis for the fundamental mode is designed based on the coupling matrix extraction and external quality factor at the input and the output resonator. The realization coupling matrix (M), and its actual matrix (m), which conforms for chosen topology is optimized and extracted $\mathrm{as}^{[20]}$.

$$
M=\left[\begin{array}{cc}
0 & 1.0201 \\
1.0201 & 0
\end{array}\right]
$$

The external quality factors are $\mathrm{q} 1=\mathrm{q} 2=0.735$ and hence, the actual external quality factors at input and output Qei=Qeo are calculated as ${ }^{[21]}$.

$$
\begin{aligned}
& F B W=\frac{B W}{f_{0}} \\
& m=F B W \times M \\
& Q=\frac{q}{F B W}
\end{aligned}
$$

The actual (denormalized) coupling matrix becomes

$$
\begin{aligned}
& m=\left[\begin{array}{cc}
0 & 0.0428 \\
0.0428 & 0
\end{array}\right] \\
& Q=17.53
\end{aligned}
$$

\section{CRLH COUPLED RESONATOR FILTER}

The coupling coefficient of the CRLH resonators is illustrated in Fig. 2. It is clear that to achieve the previous coupling coefficient $[\mathrm{m}]$ different orientation of the two resonators is investigated ${ }^{[21]}$. Therefore, the coupling 
coefficient results shown in Fig. 2 is achieved by proper orientation of a pair of identical CRLH resonators which are separated by a spacing S. The coupling coefficient which is shown in Fig. 2 is extracted using EM simulator by coupling the structure very weakly to a $50 \Omega$ microstrip feed. The coupling coefficients can be extracted from the separation frequencies ${ }^{[21]}$.

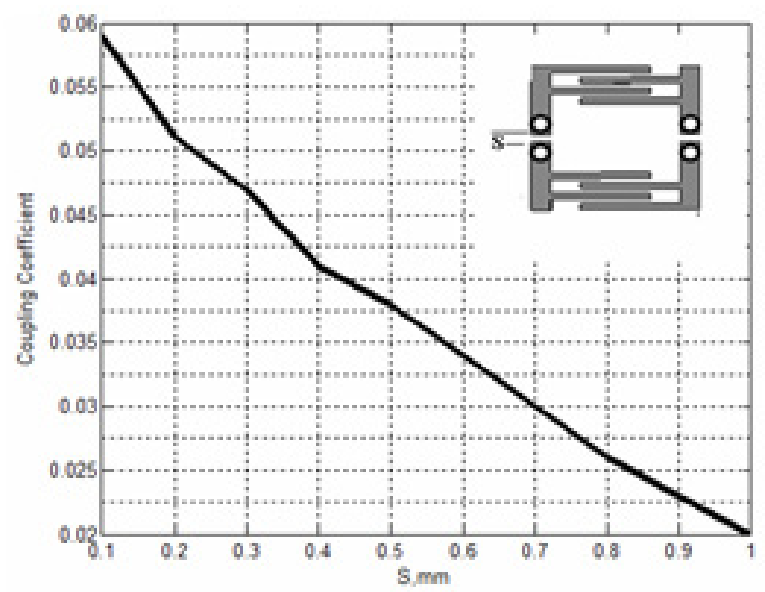

Fig. 2: Coupling coefficient for the CRLH resonators.

The CRLH coupled resonator band pass filter layout is shown in Fig. 3. As shown in the figure, the filter is designed using two couple CRLH resonators. The filter is matched to two $50 \Omega$ microstrip transmission lines at the filter ends. The microstrip filter is designed on substrate RO6010 with relative permittivity $=10.2$, a dielectric loss tangent $=0.0027$ and thickness $(\mathrm{h})=1.27 \mathrm{~mm}$. The coupling coefficient is extracted using EM simulator as shown in Fig.2. The value of the external quality factor is extracted from the full-wave EM simulator when the feed line is slightly moved distance $d$. This is illustrated in the filter layout shown in Fig. 3. According to the design procedures, the coupled resonator filter dimensions are as indicated in Fig. 3. The transmission and reflection coefficient of the CRLH filter are shown in Fig. 4. It can be seen that the filter bandwidth identified by 3 $\mathrm{dB}$ insertion loss lies between $4.67 \mathrm{GHz}$ and $4.87 \mathrm{GHz}$. Within the frequency passband the filter has almost transmission coefficient $\left(\mathrm{s}_{21}\right)$ which is close to $1.8 \mathrm{~dB}$ and reflection coefficient better than $-10 \mathrm{~dB}$ reflection coefficient. From Fig.4 it is clear that a reasonable agreement between the EM simulation and the theory depend on the optimization technique is achieved.

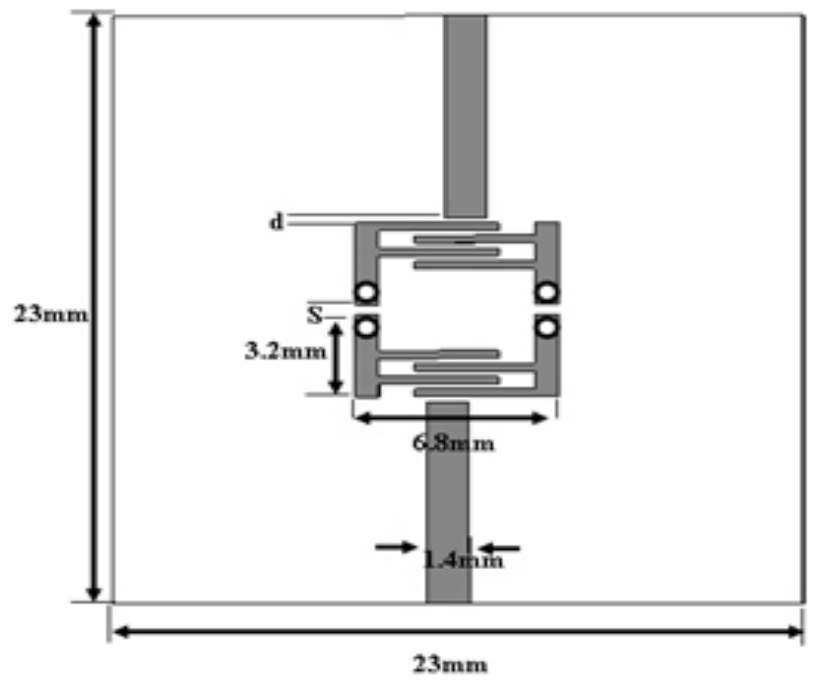

Fig. 3: 2D layout of the CRLH coupled resonator filter.

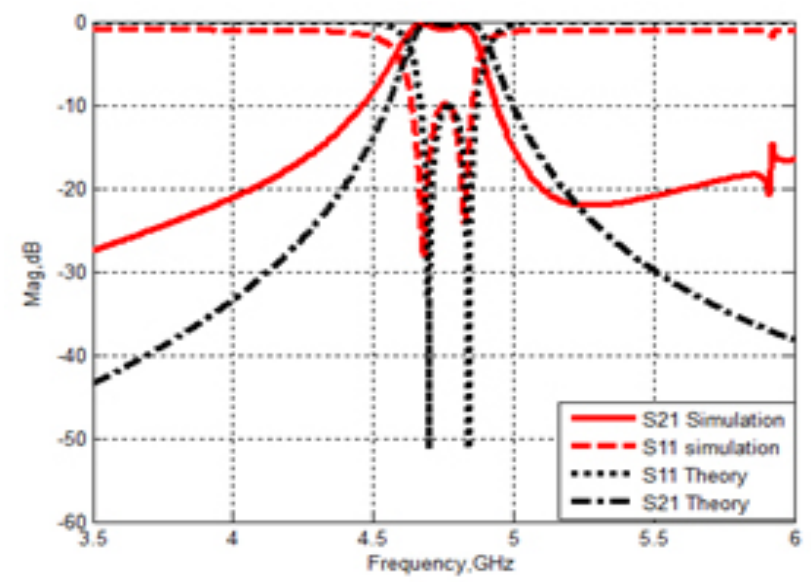

Fig. 4: CRLH coupled resonator filter response.

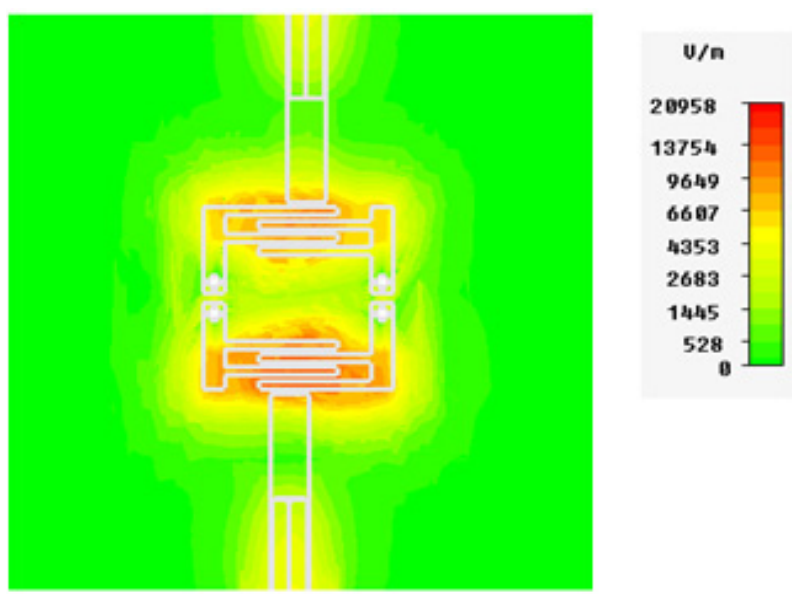

Fig. 5: Electric field distribution in the CRLH filter at $4.77 \mathrm{GHz}$. 

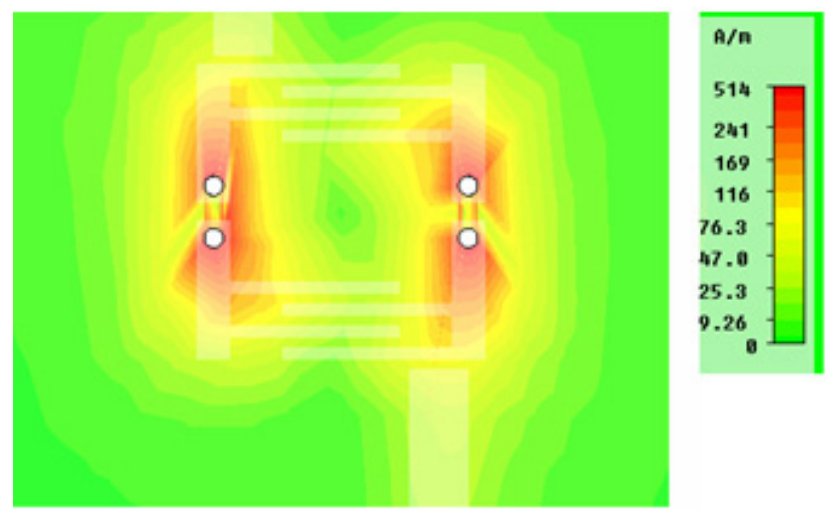

Fig. 6: Magnetic field distribution in the CRLH filter at $4.77 \mathrm{GHz}$.

The electric and magnetic field distributions at the center frequency $4.77 \mathrm{GHz}$ are shown in Fig5and Fig6 respectively. It is obvious that the electric field is concentrated around the interdigital capacitor and also the magnetic field is concentrated around the vias as seen in. From Fig.5and Fig. 6 it is concluded that the coupling in the CRLH resonators is considered mixed coupling. The fabricated photograph of the CRLH bandpass filter is shown in Fig. 7. As a final check for the designed CRLH filter, the scattering parameters of the filter were measured and compared to the simulated ones as illustrated in Fig. 8. As shown in the figure, the experimental results illustrates that the center frequency of the fabricated filter is $4.81 \mathrm{GHz}$, approximately, with almost $-10 \mathrm{~dB}$ return loss. Comparing to simulated results, we can claim that both simulated and experimental results has a good agreement between them However, there is frequency shift between the simulated and measured results can be observed. This can be as a result of the imperfect full wave simulation and the fabrication accuracy which cannot be totally avoided.

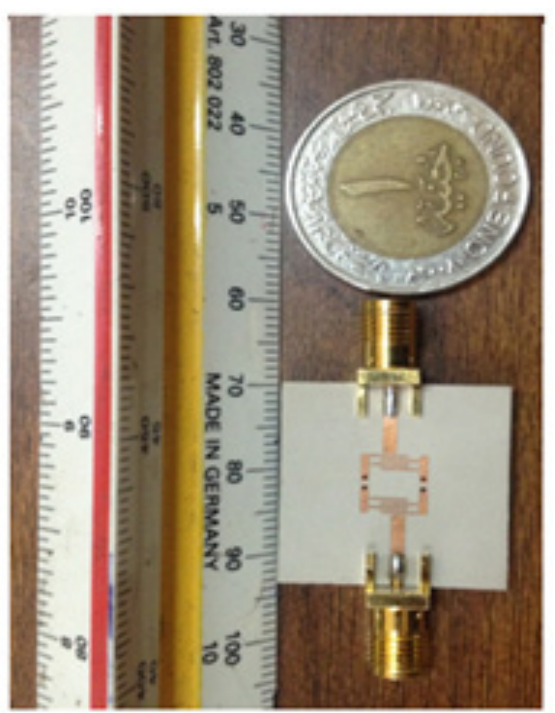

Fig. 7: A photograph of the fabricated CRLH coupled resonator filter.

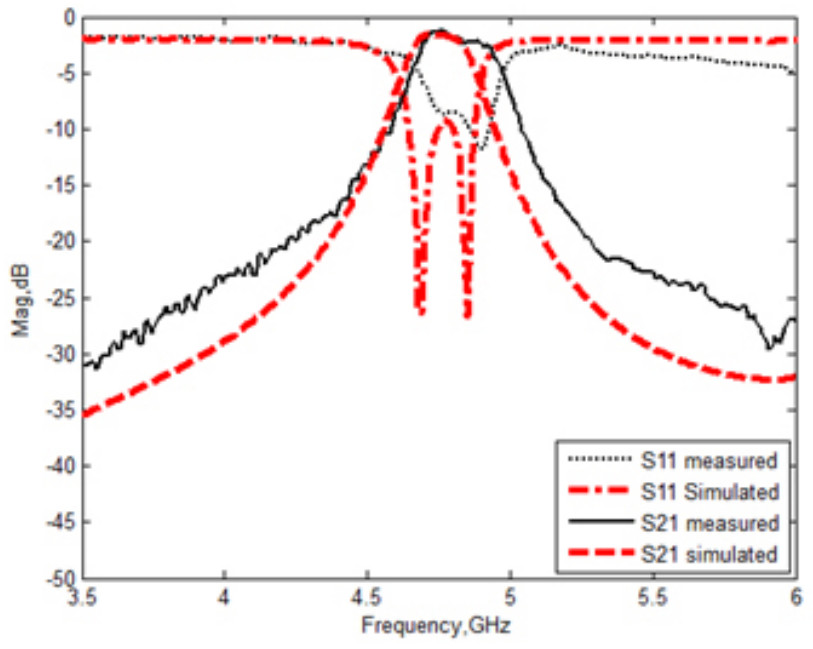

Fig. 8: Scattering parameter magnitudes of simulated and measured results of CRLH coupled resonator filter.

\section{CONCLUSION}

A microstrip compact size second order bandpass filter for wireless application has been introduced. The band pass filter is based on Composite Right/Left handed transmission line the bandpass filter has the advantage of compactness (its size is only $2.3 \times 2.3 \mathrm{~cm} 2$ ). A second order band pass filters with centre frequency of 4.77GHz and $4.8 \mathrm{GHz}$ have been designed, simulated and measured. A Good agreement between simulated and measured result has been achieved.

\section{REFERENCES}

[1] D. M. Pozar, Microwave Engineering, $4^{\text {nd }}$ ed.Wiley, 2011.

[2] Pu-Hua Deng, Jen-Tse Tsai. "Design of Microstrip Cross-Coupled Bandpass Filter With Multiple Independent Designable Transmission Zeros Using Branch-Line Resonators", IEEE Microwave and Wireless Components Letters, vol. 23, no. 5, May 2013.

[3] A. A. Oliner, "A planar negative-refractive-index medium without resonant elements," in Proc. IMS Int. Microwave Symp., Philadelphia, USA, 2003, pp. $191-194$.

[4] C. Caloz and T. Itoh, "Transmission line approach of left-handed (LH) materials and microstrip implementation of an artificial LH transmission line," IEEE Transactions on Antennas and Propagation, vol. 52, Dec 2004, pp. $1159-66$.

[5] P. L. Chi and T. Itoh, "Miniaturized dual-band directional couplers using composite right/left-handed transmission structures and their applications in beam pattern diversity systems," IEEE Trans. Microw. Theory Tech., vol. 57, no. 5, pp. 1207-1215, May 2009.

[6] M. A. Abdalla and Z. Hu, " Compact Novel CPW Ferrite Coupled Line Circulator with Left-handed Power Divider/Combiner", European Microwave Week, EuMW2011, Digest, Manchester, UK, October 9- 14, 2011, pp. 794 -707.

[7] Mahmoud A. Abdalla and Ahmed Fouad, "Integrated Filtering Antenna Based on D-CRLH Transmission Lines for Ultra-Compact Wireless Applications", Progress in electromagnetic Research C, PIER-C, vol. 66 , pp. $29-38,2016$.

[8] A. Abdalla, A., K. S. Mahmoud, "A Compact SIW Metamaterial Coupled Gap Zeroth Order Bandpass Filter with Two Transmission Zeros", $201610^{\text {th }}$ International Congress on Advanced Electromagnetic Material in Microwave and Optics, 17- 22 September 2016, Greece, pp. 4- 6.

[9] Mahmoud Abdalla, A. Y. Hassan, A. M. Galal Eldin, "A Compact High Selective Coupled Gap CRLH TL Based Bandpass Filter", 2015 $9^{\text {th }}$ International Congress on Advanced Electromagnetic Material in Microwave and Optics, 7- 12 September 2015, UK, pp. 237- 239.

[10] Mohammed. A. Fouad and Mahmoud A Abdalla, "Ultra Compact CPW Dual Band Filter Based on $\Pi$-Generalized Metamaterial NRI 
Transmission Line", Journal of Electromagnetic Waves and Applications, vol. 29, Issue 8, pp. 1093- 1103, May 2015.

[11] Mohammed. A. Fouad and Mahmoud A Abdalla, "A New $\pi-T$ Generalized Metamaterial NRI Transmission Line for a Compact CPW Triple BPF Applications", IET microwave, antenna and propagation, vol. 8, issue 13, pp. $1097-1104,2014$.

[12] Ki-Cheol Yoon, Hyun-Wook Lee, Jong-Chul Lee, Seong-Cheol Kim, "A Dual-Band Narrow Band-Pass Filter With Half-Wavelength Open Stubs Using The CRLH Metamaterial," Microwave And Optical Technology Letters, Vol. 55, No. 8, August 2013.

[13] P. L. Chi and T. Itoh, "Miniaturized dual-band directional couplers using composite right/left-handed transmission structures and their applications in beam pattern diversity systems," IEEE Trans. Microw. Theory Tech., vol. 57, no. 5, pp. 1207-1215, May 2009.

[14] X.-G. Huang, Q.-Y. Feng, Q.-Y. Xiang, and D.-H. Jia "Design of Bandpass Filter with Transmission Zeros Using Zeroth-order Resonator and U-shaped Resonator," Progress In Electromagnetics Research Symposium Proceedings, Moscow, Russia, August 19- 23, 12012.

[15] Viveka Nand Mishra, Raghvendra Kumar Chaudhary, Kumar Vaibhav Srivastava, Animesh Biswas, "Compact Two Pole Bandpass Filter Using Symmetrical Composite Right/Left Handed Transmission Line with Vias, "Proceedings of 2010 IEEE Asia-Pacific Conference on Applied Electromagnetics (APACE 2010).
[16] Viveka Nand Mishra, Raghvendra Kumar Chaudhary, Kumar Vaibhav Srivastava, Animesh Biswas, "Compact Two Pole Bandpass Filter Implemented Using Via-free Composite Right/Left Handed Transmission Line with Radial Stubs," Proceedings of the $41^{\text {st }}$ European Microwave Conference 2011.

[17] C. Caloz and T. Itoh, Electromagnetic Metamaterials Transmission Line Theory and Microwave Applications. New Jersey: John Wiey \& Sons, 2006.

[18] M. A. Abdalla; A. Y. Hassan; A. M. Galal Eldin, "A compact high selective coupled gap CRLH TL based bandpass filter," $20159^{\text {th }}$ International Congress on Advanced Electromagnetic Materials in Microwaves and Optics (METAMATERIALS).

[19] Jakub Sorocki; Ilona Piekarz; Krzysztof Wincza; Slawomir Gruszczynski, "Right/Left-Handed Transmission Lines Based on Coupled Transmission Line Sections and Their Application Towards Bandpass Filters, "IEEE Transactions on Microwave Theory and Techniques Year: 2015, Volume: 63, Issue: 2 Pages: 384 - 396.

[20] S. Amari, "Synthesis of Cross-Coupled Resonator Filters Using an Analytical Gradiant-Based Optimi-zation Technique", IEEE Trans. Microwave. Theory Tech., vol. 48, No. 9, pp. 1559- 1564, Sep. 2000. [21] Hong, J. S. and M. J. Lancaster, Microstrip Filters for RF/ Microwave Applications, Wiley, New York, 2001. 